DOI : $10.24850 / j-t y c a-2021-05-10$

Notas

\title{
Aquappolis: aplicación móvil para la captación de agua de Iluvia en la CDMX
}

\section{Aquappolis: Mobile application for rainwater harvesting in Mexico City}

Carlos Sánchez-Vargas ${ }^{1}$

José Luis Salinas-Estévez²

${ }^{1}$ Instituto de Ingeniería, Universidad Nacional Autónoma de México, Ciudad de México, México, CSanchezV@iingen.unam.mx

2Instituto de Ingeniería, Universidad Nacional Autónoma de México, Ciudad de México, México, JSalinasE@iingen.unam.mx

Autor para correspondencia: José Luis Salinas-Estévez, JSalinasE@iingen.unam.mx

\section{Resumen}

La captación de agua pluvial se ha convertido en una acción indispensable alrededor del mundo para satisfacer las necesidades de grandes ciudades donde el acceso a este recurso es limitado (Shivakumar, 2017), tal es el 
caso de la Ciudad de México, donde la demanda rebasa la capacidad de las fuentes de abastecimiento y donde además se presentan diversos problemas relacionados con el uso del agua (Arto-Olaizola et al., 2016). Aquappolis es una aplicación móvil destinada a calcular la cantidad de agua de lluvia que puede ser captada en una determinada zona. La información que usa esta aplicación es obtenida de los datos recaudados por la amplia red de sensores de las estaciones del Observatorio Hidrológico del Instituto de Ingeniería (II-UNAM) en la Ciudad de México. En este trabajo se proporciona una muestra de cómo la innovación tecnológica y la información instantánea puesta al alcance de las autoridades encargadas de la toma de decisiones, así como de la población en general, son indispensables para lograr un manejo responsable, cuidadoso y sustentable de los recursos hídricos disponibles en la Zona Metropolitana del Valle de México.

Palabras clave: tecnología, innovación, aplicación móvil, smartphone, captación de agua, lluvia, gestión del agua.

\section{Abstract}

Rainwater harvesting has become a necessary action around the world to satisfy the big cities needs where the water access is restrained (Shivakumar, 2017), which is the case of Mexico City where the water demand exceeds the supply sources, and many other problems are related to water (Arto-Olaizola et al., 2016). Aquappolis is a mobile application intended to compute the amount of rainwater that can be harvested from specific zones in Mexico City. The app information is 
provided from extensive rain gauge network data collected from Hydrological Observatory stations across Mexico City. This paper provides an example of how the technological innovation and instant information available for decision maker authorities and general population are indispensable to achieve a responsible, careful, and sustainable management of available water resources in Mexico's Valley.

Keywords: Technology, innovation, application mobile, smartphone, rainwater harvesting, rainfall, water management.

Recibido: 08/01/2020

Aceptado: 08/10/2020

\section{Introducción}

En épocas recientes, la Ciudad de México ha presentado una problemática considerable respecto al manejo del agua. Inundaciones, escasez de agua y un rebase de la capacidad del sistema de drenaje en ciertas temporadas del año son cada vez más frecuentes e impactan en las actividades de la ciudad y en la calidad de vida de sus habitantes, propiciando situaciones de riesgo para la población. 
La Ciudad de México fue asentada hace siglos sobre lo que era en ese entonces un sistema de lagos interconectados: Texcoco, Xochimilco, Chalco y Zumpango, que ocupaban un área aproximada de 114 mil hectáreas, como se muestra en la Figura 1. Estos lagos eran abastecidos por aguas de una gran cantidad de ríos provenientes de las cadenas montañosas circundantes. El Eje Neovolcánico, que cruza todo el país desde el Golfo de México hasta el océano Pacifico, generó la topografía montañosa en la región; asimismo, las constantes emanaciones volcánicas proveyeron de material arcilloso a la zona y se acumuló en el lecho de los lagos. 
Tecnología y

Ciencias $\approx$ Agua
2021, Instituto Mexicano de Tecnología del Agua

Open Access bajo la licencia CC BY-NC-SA 4.0

(https://creativecommons.org/licenses/by-nc-sa/4.0/)

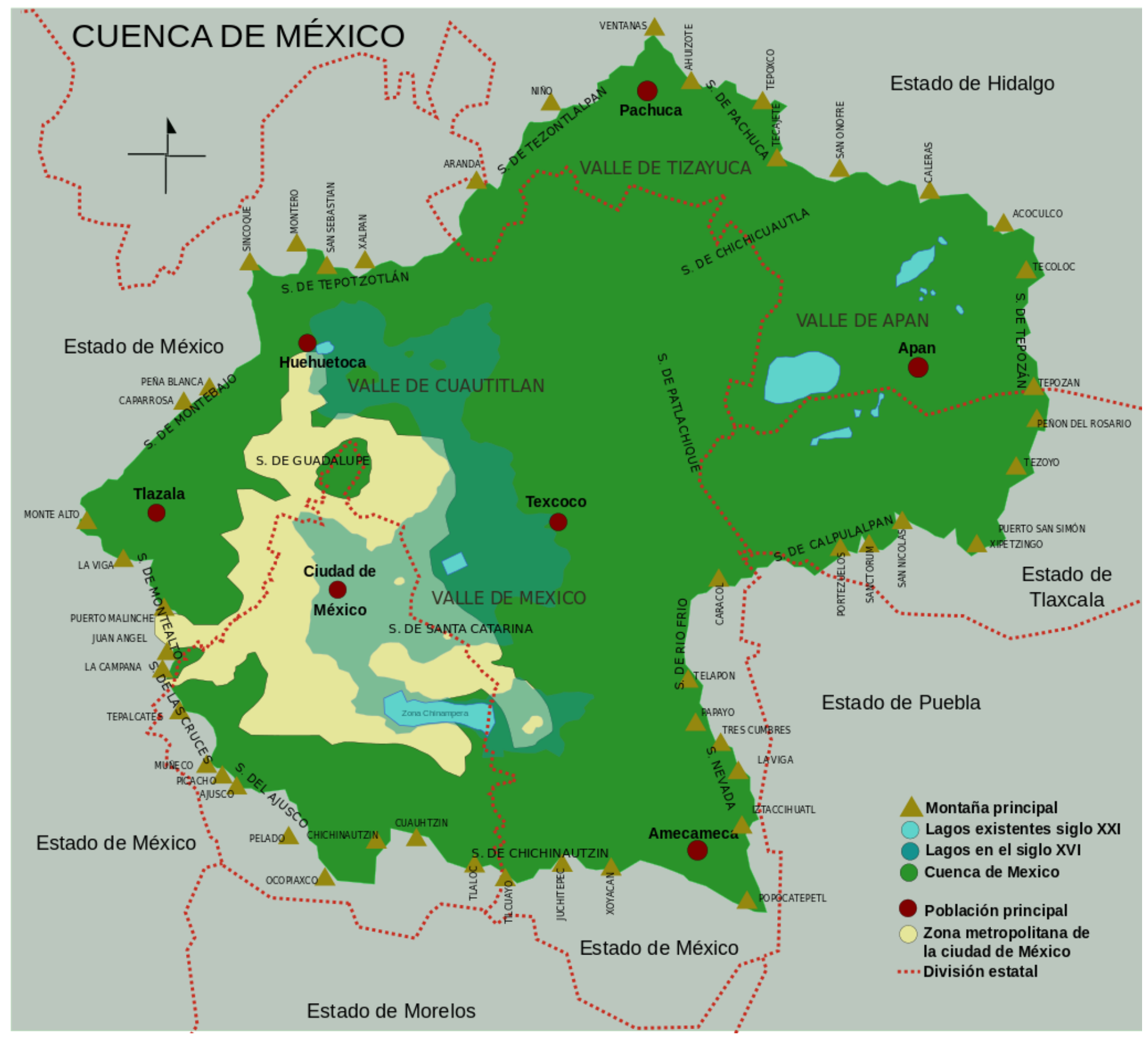

Figura 1. Antiguo sistema lacustre de la cuenca de México. 
La antigua ciudad de Tenochtitlan se asentó sobre un islote del lago de Texcoco y desarrolló su agricultura mediante chinampas, suelo artificial para cultivo sobre el agua. También contaba con un sistema de diques que permitían controlar el nivel variable del lago, para así evitar inundaciones; como función adicional tenía separarel agua dulce del agua salada. A partir de la época colonial, los lagos del Valle de México experimentaron un proceso de desecación con el fin de evitar inundaciones, sin embargo, seguían ocurriendo, por lo que durante el Porfiriato comenzó la construcción de un sistema de drenaje de aguas negras y de agua pluvial que tenía la función de llevar tales líquidos al estado de Hidalgo. Con el paso del tiempo, por la urbanización y los asentamientos en la ciudad, el volumen de aguas a evacuar cada año fue creciendo, por lo que el sistema de drenaje se vio rebasado en su capacidad y surgió la necesidad de crear un nuevo sistema de avenamiento capaz de evacuar esos grandes volúmenes cuando fuera necesario.

En los últimos cien años, la población de la Ciudad de México creció de manera abrupta. De 0.7 millones de personas en 1900, la población pasó a 8.9 millones en 2015, según el último censo del Instituto Nacional de Estadística y Geografía (INEGI) (Figura 2 y Figura 3) (INEGI, 2015a). El periodo donde se registró un mayor aumento en la población fue de 1920 a 1970, con un crecimiento de 5.7 millones. Actualmente, si se cuenta la población del área conurbada, el número de habitantes es de 22 millones, lo que ubica a la zona como la aglomeración poblacional más grande del continente americano. Debido a esta gran cantidad de 
Tecnología y

Ciencias $₫$ Agua
2021, Instituto Mexicano de Tecnología del Agua

Open Access bajo la licencia CC BY-NC-SA 4.0

(https://creativecommons.org/licenses/by-nc-sa/4.0/)

personas, satisfacer la demanda de 68 metros cúbicos por segundo de agua se vuelve un gran reto para el Gobierno de la Ciudad de México.

Crecimiento poblacional CDMX

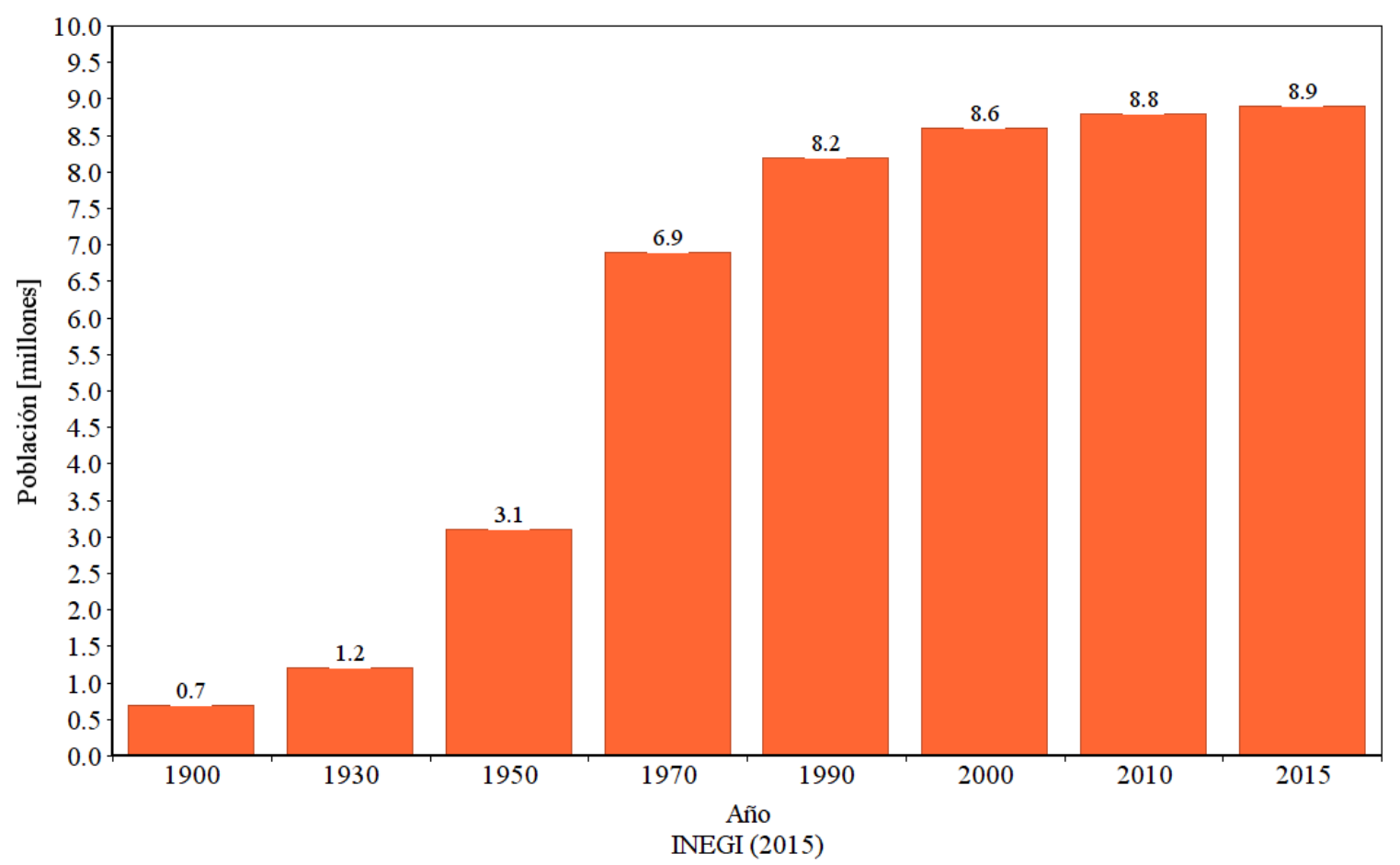

Figura 2. Crecimiento poblacional en la Ciudad de México (1900-2015). 
Tecnología y

Ciencias $₫$ Agua
2021, Instituto Mexicano de Tecnología del Agua

Open Access bajo la licencia CC BY-NC-SA 4.0

(https://creativecommons.org/licenses/by-nc-sa/4.0/)

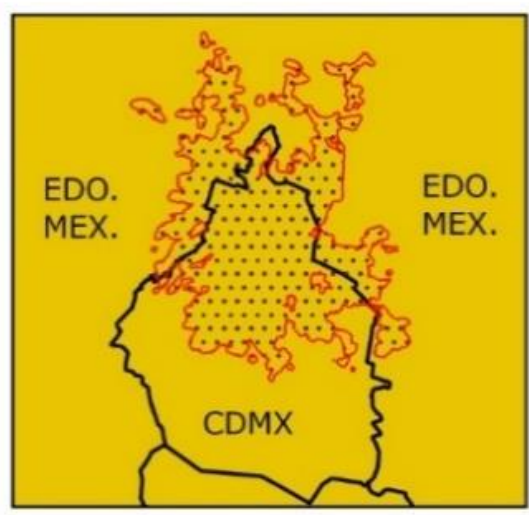

1900

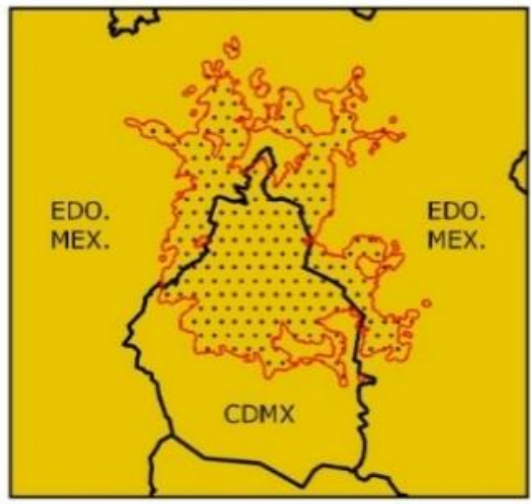

2000

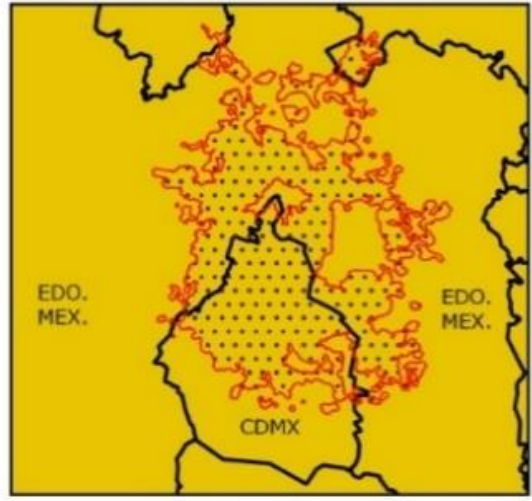

2014

Figura 3. Crecimiento de la mancha urbana (1900-2014).

Debido a la fuerte demanda de agua, la ciudad debe importar este vital líquido desde las cuencas aledañas de Cutzamala y Lerma, que aportan un poco más del 30 \% de la cantidad requerida (Conagua, 2005), el resto se obtiene de pozos distribuidos en el área de la cuenca. El hecho de que la ciudad esté cubierta mayoritariamente por concreto, la falta de áreas verdes y la existencia de un suelo arcilloso que permite la infiltración a velocidades muy bajas, aunado a la gran cantidad de agua extraída, provoca un déficit importante para los acuíferos; esto, a su vez, ha ocasionado asentamientos sobre la parte de la ciudad ubicada sobre el lecho del lago de hasta 10 metros en los últimos 60 años en algunos puntos (De Urbanisten, Deltares, 2017).

La ciudad sufre dos situaciones contradictorias: escasez de agua potable y exceso de agua en temporada de Iluvias. El ciudadano promedio en la CDMX consume unos 366 litros de agua al día, cantidad que rebasa por mucho el consumo recomendado por la Organización Mundial de la 
Teçnología y

Ciencias $₫$ Agua
2021, Instituto Mexicano de Tecnología del Agua

Open Access bajo la licencia CC BY-NC-SA 4.0

(https://creativecommons.org/licenses/by-nc-sa/4.0/)

Salud (OMS) de 80 litros (OMS, 2003). Esta gran demanda ocasiona que cada año se aumenten los volúmenes de extracción de los pozos, acelerando la disminución del nivel de agua en los acuíferos e incrementando los asentamientos que, día a día, provocan daños a la infraestructura de la ciudad, en especial de aquella que abastece de agua a los usuarios de la red, ocasionando que alrededor del $35 \%$ del agua que transporta la tubería se pierda en fugas (Sacmex, 2017). Las 16 alcaldías de la CDMX se ven afectadas por la escasez de agua cuando hay cortes en el abastecimiento, pero en especial Iztapalapa y Tláhuac, donde el consumo promedio baja a 230 litros al día por habitante (Sedema, 2016) (Tabla 1) y el abasto se da a través de tandeos, como se muestra en la Figura 4.

Tabla 1. Tabla de población y dotación por alcaldía de la CDMX.

\begin{tabular}{|c|c|c|}
\hline Alcaldía & Población & Dotación (I/hab/día) \\
\hline Iztapalapa & 1783535 & 235 \\
\hline Gustavo A. Madero & 1242676 & 321 \\
\hline Álvaro Obregón & 690568 & 355 \\
\hline Coyoacán & 643838 & 560 \\
\hline Tlalpan & 584992 & 332 \\
\hline Cuauhtémoc & 519224 & 203 \\
\hline Venustiano Carranza & 465571 & 404 \\
\hline Azcapotzalco & 443071 & \\
\hline
\end{tabular}


Tecnología y

Ciencias $\cong$ Agua
2021, Instituto Mexicano de Tecnología del Agua

Open Access bajo la licencia CC BY-NC-SA 4.0

(https://creativecommons.org/licenses/by-nc-sa/4.0/)

\begin{tabular}{|c|c|c|}
\hline Iztacalco & 413649 & 219 \\
\hline Xochimilco & 372111 & 374 \\
\hline Benito Juárez & 362591 & 406 \\
\hline Miguel Hidalgo & 354803 & 502 \\
\hline Tláhuac & 304611 & 210 \\
\hline Magdalena Contreras & 223266 & 554 \\
\hline Cuajimalpa & 152306 & 293 \\
\hline Milpa Alta & 96922 & 410 \\
\hline
\end{tabular}

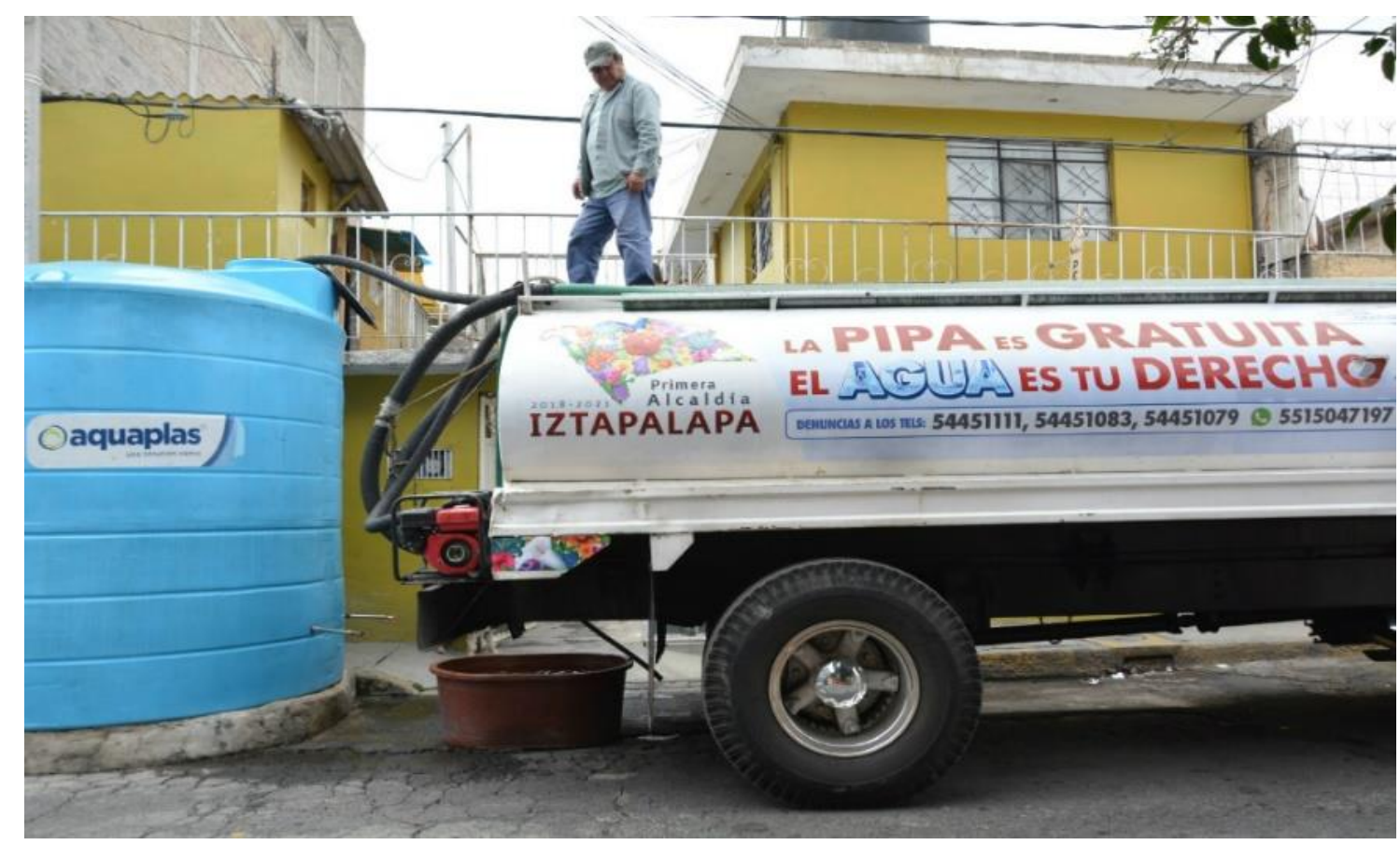

Figura 4. Ante el desabasto de agua potable debido al corte en el suministro, el gobierno de la CDMX pone a disposición de la ciudadanía pipas para dotar de líquido a la población de las zonas afectadas. 
La frecuencia de las inundaciones urbanas ha aumentado, pues en las zonas montañosas del sur y suroeste de la ciudad, el avance de la mancha urbana ha deforestado y pavimentado gran parte de lo que antes era bosque y zonas de infiltración, ocasionando que en los eventos de Iluvia las escorrentías fluyan a grandes velocidades, situación que impide que el agua se infiltre durante su recorrido y que al llegar a las zonas bajas se acumule y provoque encharcamientos e inundaciones (Figura 5). Cuando ello sucede, el sistema de drenajes se llega a ver sobrepasado no solamente por la cantidad de agua precipitada, sino también por el acarreo de basura que obstruye el ingreso del agua al sistema.

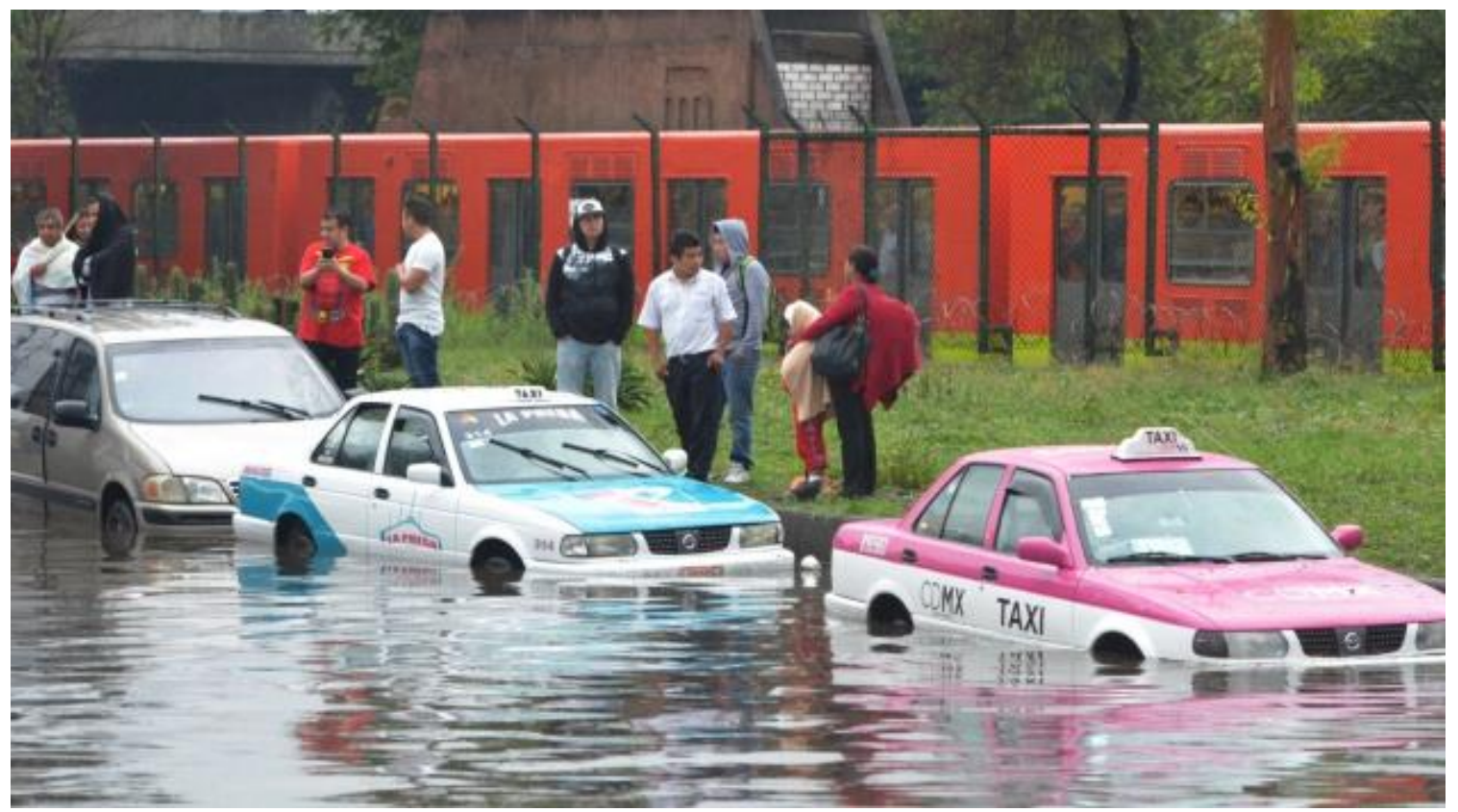

Figura 5. Durante la temporada de lluvias, las zonas vulnerables a inundación se ven afectadas y generan problemas para la movilidad. 
En cuanto a la acción política ante esta problemática, los principales actores gubernamentales han entrado recientemente en roces entre ellos y las instancias mediadoras no han puesto fin a los desacuerdos para que los gobiernos trabajen en conjunto. La CDMX, antes Distrito Federal, era una dependencia del Gobierno Federal, por lo que se vio favorecida en cuanto a la construcción de infraestructura para abastecimiento de agua potable y desalojo de aguas negras; posterior a su reforma política apareció la necesidad de relacionarse con los estados aledaños, principalmente el Estado de México (Edomex), a fin de tener la capacidad de negociar el agua necesaria para satisfacerla demanda de la población. Si bien la CDMX paga de forma puntual los volúmenes de agua que le son suministrados, no se debe continuar con la importación de agua a gran escala, aunque siga siendo indispensable para satisfacer la demanda.

En cuanto al Estado de México, demanda que se tome en consideración un presupuesto del Gobierno Federal destinado a la recuperación de los mantos acuíferos; también exige que el Gobierno de la Ciudad de México tome responsabilidad para resarcir las afectaciones ecológicas e hidrológicas que se han ocasionado durante el tiempo que se ha exportado agua hacia el área metropolitana. Asimismo, es necesario remarcar que el Edomex se ha visto favorecido por la infraestructura que se le dio a la CDMX que también abastece a sus municipios aledaños a esta entidad.

Ambas entidades deben colaborar en conjunto y con los estados que se ven recientemente incluidas en esta situación, como Hidalgo y 
Michoacán, junto con la intervención del Gobierno Federal, por conducto de la Conagua, para darle solución a los problemas que aquejan a la Zona Metropolitana del Valle de México

Es insuficiente adoptar como única solución la construcción de grandes obras de infraestructura, así como la ampliación de los sistemas actuales, la importación de agua de otras zonas o mayor explotación de los acuíferos con los que se cuentan, pues llevar a cabo estas acciones conlleva un gran desafío político, social, económico y ecológico; por lo tanto, la limitación de las soluciones lleva a considerar alternativas que son contrarias a la forma tradicional de las políticas hidráulicas (PerlóCohen \& González-Reynoso, 2005).

En la Zona Metropolitana del Valle de México se requiere adoptar un manejo integral del agua para satisfacer las necesidades de la población actual y futura. Es de suma importancia erradicar el estrés hídrico al que se ve sometida la cuenca; reducir el consumo per cápita de agua; diseñar mejores políticas públicas sobre su manejo; disminuir las fugas de los sistemas de abastecimiento; incentivar el aprovechamiento de agua tratada para uso agrícola; incrementar la cantidad de áreas verdes; tener mayor regulación sobre la urbanización; y fomentar la conciencia de la población sobre un consumo responsable del agua para conservar, cuidar y aprovechar este líquido. Llevar a cabo estas acciones puede reducir de manera significativa la problemática presente en la actualidad.

Así, la recolección de agua de lluvia en domicilios aparece como una opción muy viable tanto para los usuarios como para las dependencias responsables de abastecer agua para consumo humano. La Iluvia en la 
CDMX no es escasa; en la temporada de lluvias aporta grandes volúmenes que no son aprovechados y que son enviados directamente a los sistemas de drenaje. Los sistemas de captación de agua de Iluvia funcionan de manera sencilla: el agua que cae sobre los techos es captada y se conduce por un sistema de tubos hasta un filtro que la purifica para finalmente ser almacenada en un tanque para su disposición y con ello disminuir el consumo directo de la red.

En México existen proyectos que tienen como objetivo distribuir el agua de forma equitativa sin explotar los acuíferos presentes en la zona; esto se hace con el aprovechamiento de la lluvia, un recurso que se desperdicia en el drenaje y que en la CDMX durante 2018 presentó un promedio de $714 \mathrm{~mm}$ de Iluvia (Semarnat, 2018).

Isla Urbana es una organización que se dedica a la implementación de proyectos dedicados a la captación del agua de lluvia. Desde sus inicios, en 2009, ha instalado un total de 15000 sistemas para captación con 90000 usuarios que han recolectado 600 millones de litros cada año. En la CDMX se han instalado 7134 sistemas para 49938 beneficiarios (Isla Urbana, 2018).

Debido al interés de conocer el comportamiento del ciclo hidrológico urbano, en particular la intensidad y variabilidad espacial de la lluvia en la CDMX, en 2016 se creó el Observatorio Hidrológico (OH) del Instituto de Ingeniería de la UNAM; se trata de un proyecto que tiene por objetivo proporcionar información en tiempo real sobre la precipitación en la Ciudad de México mediante un sistema de equipos de medición que ponen en cuestión de minutos a disposición de la población en general y de las 
personas encargadas de la toma de decisiones los datos de dónde y con qué intensidad se presenta la lluvia. El Observatorio cuenta con 55 estaciones de medición, distribuidas en el Valle de México; en su mayoría se conforman por un disdrómetro, que cuenta con un láser que realiza el conteo de número de gotas que cruzan el sensor; también mide el diámetro de las gotas y la velocidad de las mismas.

Cada una de las estaciones funciona con la ayuda de una microcomputadora de bajo costo que recibe la información medida; luego la almacena en la nube, donde es procesada, para finalmente ser mostrada en el mapa de precipitaciones del OH y ser publicada en Twitter. El sistema cuenta con una celda solar y una batería que tienen como objetivo brindar autonomía energética. Algunas de las estaciones que no cuentan con el disdrómetro poseen otro sensor de medición: un pluviómetro de pesaje. Este instrumento no puede llevar a cabo todas las mediciones que un disdrómetro realiza, no obstante sí puede determinar la intensidad de la lluvia. El observatorio contempla la colocación de más estaciones de medición, con el objetivo de incrementar su densidad en la ciudad (OH-II UNAM, 2016) (Figura 6). 
Tecnología y
2021, Instituto Mexicano de Tecnología del Agua

Open Access bajo la licencia CC BY-NC-SA 4.0

(https://creativecommons.org/licenses/by-nc-sa/4.0/)
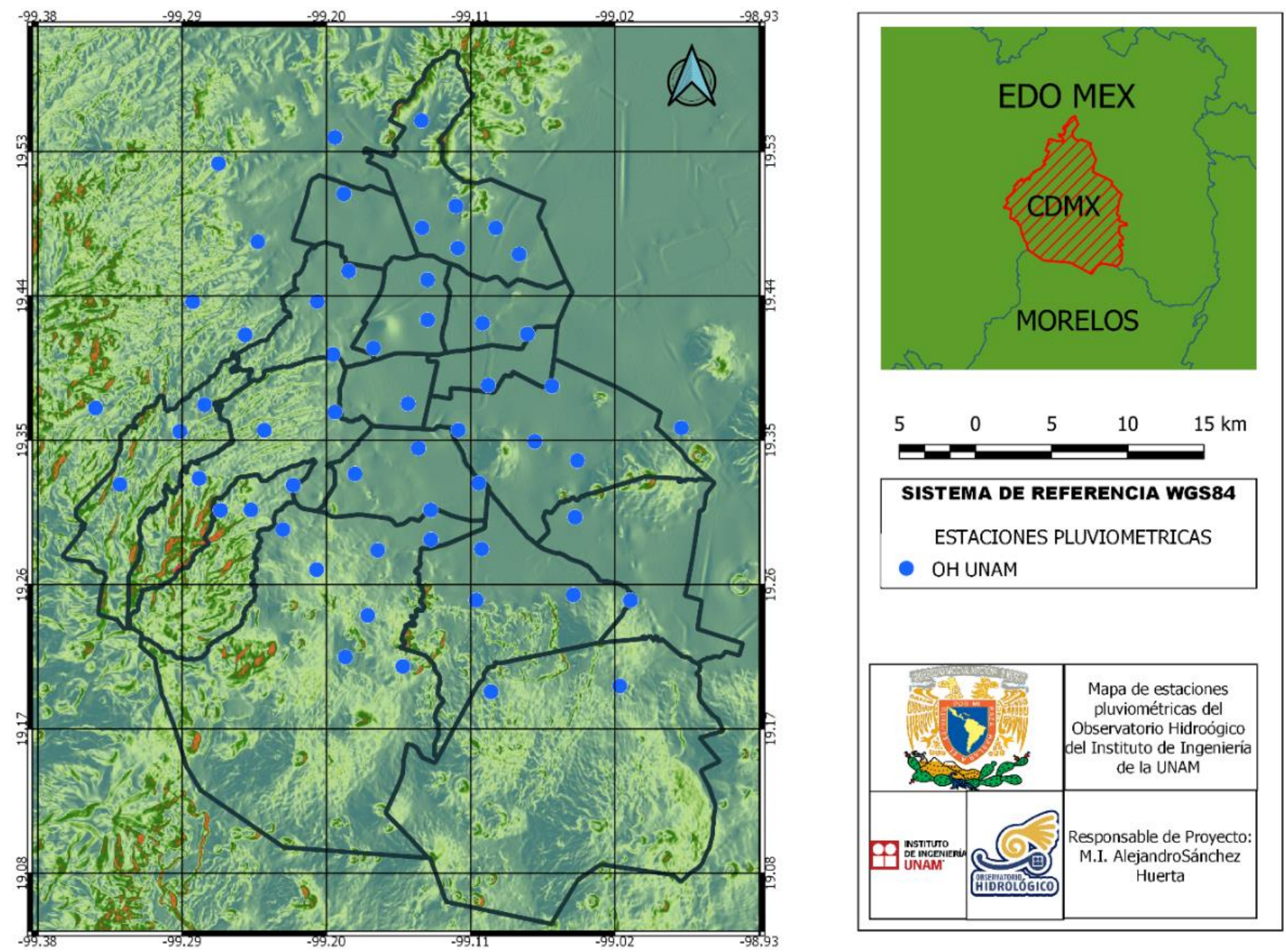

Figura 6. Mapa de ubicación de las estaciones pluviométricas del Observatorio Hidrológico II UNAM.

El Observatorio Hidrológico del Instituto de Ingeniería de la UNAM, en colaboración con Isla Urbana y el desarrollador ABEHA, desarrolló la aplicación Aquappolis, que tiene por objetivo ser la primera aplicación móvil para generación y el uso de información relacionada con el agua 
pluvial de la Ciudad de México. Esta aplicación proporciona datos del agua de Iluvia potencialmente acumulable en un área dada y en un periodo de tiempo propuesto usando los datos de precipitación medidos por los equipos del Observatorio Hidrológico ubicados a lo largo de la ciudad. Este documento tiene por objetivo mostrar el impacto positivo que la recolección de lluvia puede tener para la situación hídrica en la Ciudad de México.

\section{Materiales y métodos}

Los disdrómetros presentes en las estaciones del OH-II UNAM registran las características de cada evento de lluvia a través de un láser que proviene de un emisor y llega a un receptor, el cual mide las diferencias de voltaje cuando las partículas cruzan por él, obteniendo así de manera indirecta los diámetros de las gotas que pasan por el sensor. Para registrar la velocidad se determina el tiempo que tarda cada partícula desde que incide en el láser hasta cruzar totalmente el sensor. Para cada evento de lluvia se registran los datos de diámetro y tamaño para cada partícula; se clasifican en 32 grupos, donde las gotas van desde pequeñas a grandes, o de lentas a rápidas. 
Posteriormente, con ayuda de los datos ya recolectados, se realizan los cálculos para obtener intensidad, energía cinética, reflectividad, visibilidad y espectro a intervalos de un minuto para cada evento. Estos datos pueden verse afectados por diferentes causas, como incertidumbres instrumentales (alineación óptica, intensidad del haz de luz y distorsión por viento); muestreo (debido a los pequeños volúmenes de muestreo ocupados y que la distribución de tamaño de gotas dependa de estos volúmenes), y observación (ocasionado por las diferentes velocidades de las gotas, relacionadas con su tamaño; las partículas que comienzan su caída en un mismo punto alcanzan el suelo a diferente tiempo y distancia horizontal que puede ser de hasta kilómetros). Tales incertidumbres se tratan de disminuir al aumentar la densidad de los equipos de medición dentro del área del Valle de México y al calibra los sensores de forma adecuada.

Una vez empezada la recolección de los datos de precipitación para toda la ciudad nace la problemática de cómo ofrecer esta información a los usuarios domésticos de la red de agua para que puedan cuantificar el volumen de líquido aprovechable dependiendo de su área de captación. $\mathrm{Si}$ estos datos se muestran de manera sencilla, los usuarios pueden manifestar el deseo de disminuir los gastos asociados con el servicio de dotación de agua potable por parte del Gobierno de la Ciudad de México y también mostrar cierto grado de independencia al servicio brindado por el gobierno. Mediante la creación de una aplicación para dispositivos móviles se ponen al alcance de la ciudadanía datos confiables de volúmenes de captación (Silva et al., 2018). 
La aplicación Aquappolis trabaja con dos API (del inglés Application Programming Interface): ABEHA y $\mathrm{OH}$. La API del $\mathrm{OH}$ contiene la información de precipitación, intensidad, velocidad y distribución del tamaño de las gotas precipitadas para la CDMX dentro de una malla cuadriculada de 750 metros de espaciamiento; la API de ABEHA recibe la información del $\mathrm{OH}$ y la procesa para el usuario.

La aplicación utiliza el sistema de navegación GPS del dispositivo móvil para ubicar la zona donde se localiza el usuario y en este sitio, o en algún otro que proponga la persona, colocar un sistema de captación de agua de lluvia que dependa del área disponible, es decir, del área libre con la que cuenta el usuario.

Utilizando el área, Iluvia precipitada y un factor ajustador se puede estimar el volumen de agua que se puede captar. La aplicación también cuenta con un seleccionador de fechas, herramienta que permite seleccionar un día específico o un rango de fechas para mostrar el volumen que se puede aprovechar con base en estimaciones estadísticas.

La aplicación también permite al usuario, utilizando la cámara de su dispositivo móvil, capturar imágenes de agua estancada para que se pueda hacer un cálculo de su volumen y tener datos estadísticos de los encharcamientos en la ciudad y de zonas susceptibles (Figura 7). 


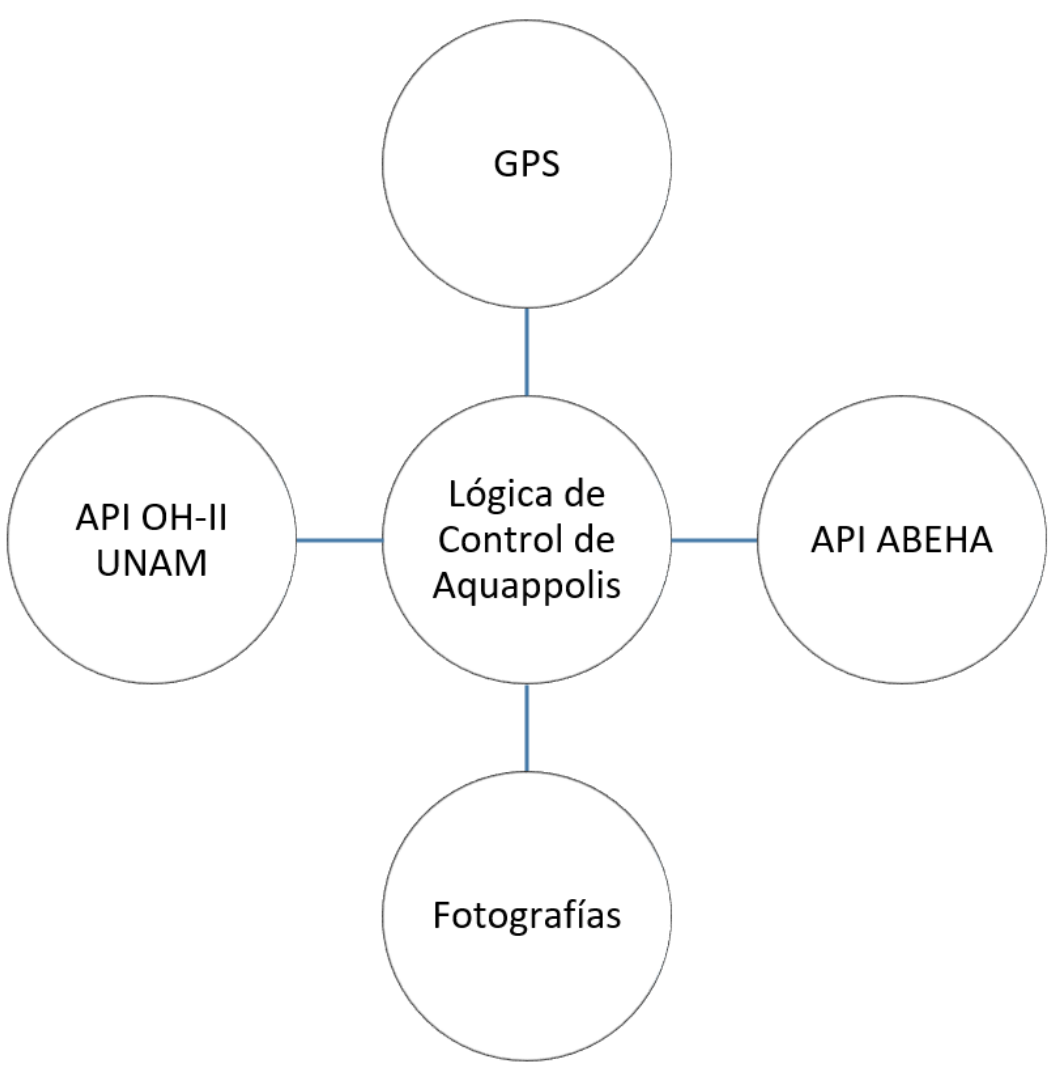

Figura 7. Esquema de arquitectura de alto nivel de Aquappolis.

De esta manera, si un usuario quiere medir el volumen del agua precipitada sobre un área disponible, primero tiene que proporcionar la ubicación del lugar (coordenadas de longitud y latitud dadas por el dispositivo) y las dimensiones del área de captación; así, la API ABEHA ubica la coordenada proporcionada en la malla de la API OH y toma la información asociada con el cuadro donde se ubica el usuario; posteriormente, con las características del sistema, obtiene el volumen para las fechas seleccionadas, y finalmente la información se presenta al usuario por la aplicación. 
Para la realización del estudio se analizaron dos puntos importantes en la CDMX ubicados en las alcaldías de Iztapalapa y Cuajimalpa debido a su condición hídrica. En la primera alcaldía el desabasto de agua es constante, mientras que en la segunda existen puntos importantes de encharcamientos e inundaciones. En ambos casos se ve el impacto que podría tener para estas zonas la captación de agua. En el primer caso se desea saber si el volumen que puede ser captado funciona como un complemento del agua potable. Según datos de Isla Urbana, la captación de agua de lluvia en la Ciudad de México podría significar para sus usuarios un ahorro de 5 a 8 meses de consumo de la red pública para un domicilio familiar. Para la zona de Cuajimalpa no se analiza el desabasto de agua; colocar un sistema de captación en esta zona podría significar un amortiguamiento, junto con infraestructura pública, para la escorrentía y acumulación de agua en la vía pública.

\section{Resultados}

La aplicación Aquappolis cumple con el objetivo de extraer y mostrar al usuario, para cada punto seleccionado dentro del área de medición de los equipos, el volumen de agua precipitado en el intervalo de tiempo seleccionado (los datos sólo se muestran para fechas posteriores a la de 
inicio de operación de la API ABEHA), tomando como dato el área disponible que recibirá toda el agua de Iluvia.

La aplicación Aquappolis requiere ciertos datos para su operación. Para tener acceso, primero se necesita contar con un usuario o bien iniciar como usuario libre; después se deben especificar las dimensiones, largo y ancho del área donde se va a realizar el análisis. Para este caso, se va a suponer un área de $12 \mathrm{~m}$ de largo por $5 \mathrm{~m}$ de ancho (Figura 8). 


\section{$\leftarrow \quad$ MI SISTEMA \\ SELECCIONA SI YA TIENES UN \\ SISTEMA DE CAPTACIÓN DE AGUA DE \\ LLUVIA O TE INTERESA UNO}

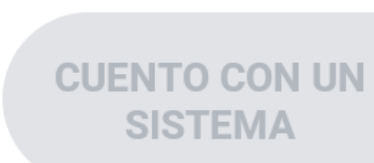
ME INTERESA
INSTALAR UNO

MEDIDAS DEL TECHO DONDE ESTARÁ

EL SISTEMA

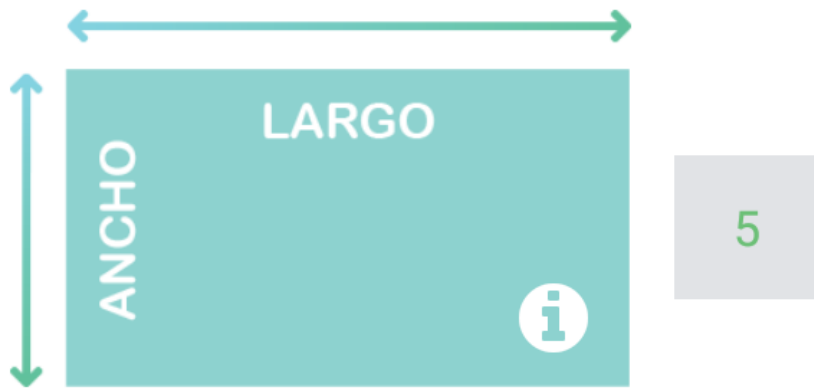

12

\section{ACEPTAR}

Figura 8. Área disponible para la captación. 
El siguiente dato por proporcionar es la ubicación geográfica del área de estudio. Se escogió un punto en la colonia Las Américas, alcaldía Iztapalapa (Figura 9), y uno en Lomas de Santa Fe, alcaldía Cuajimalpa (Figura 10).

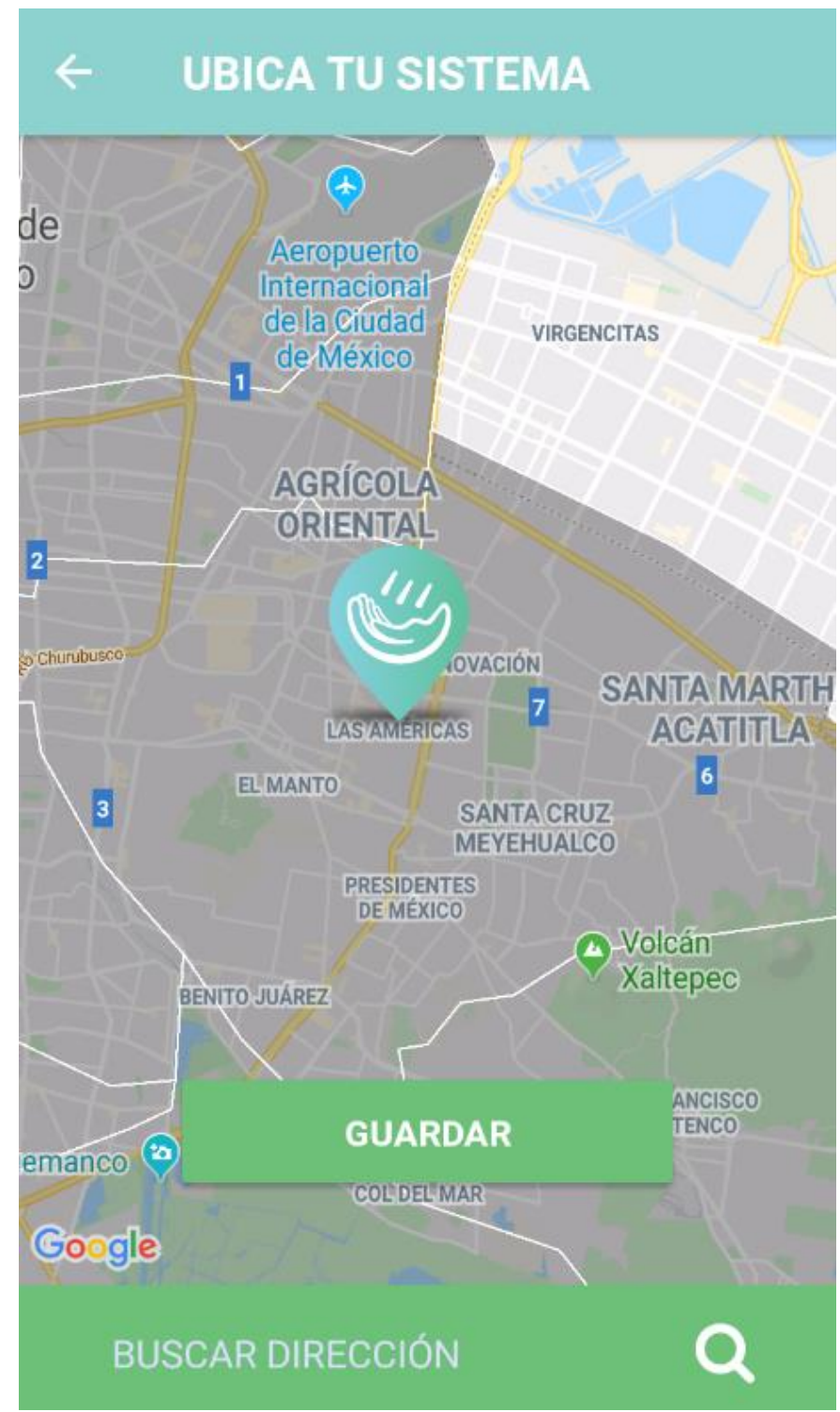

Figura 9. Ubicación del sistema de captación en la alcaldía Iztapalapa. 


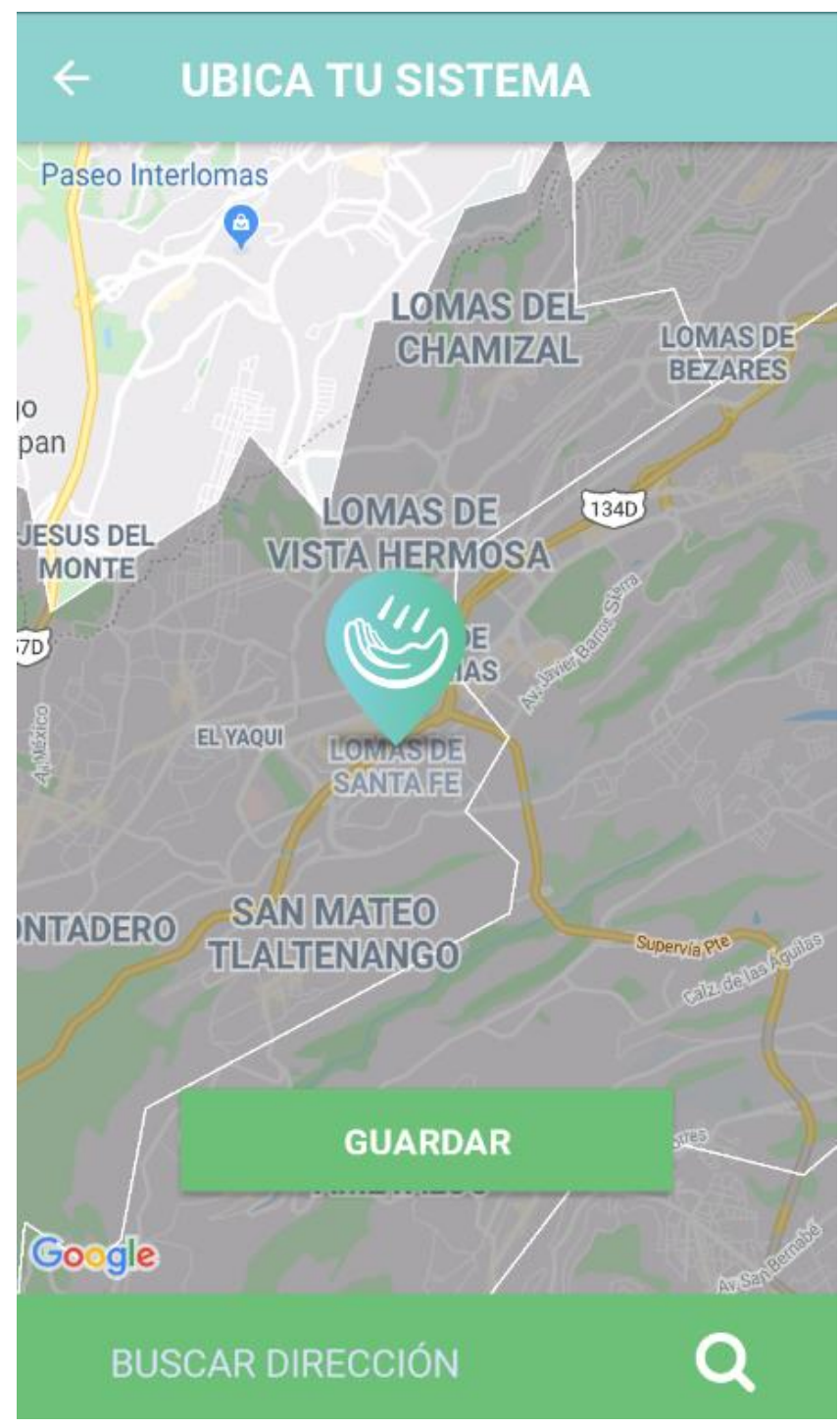

Figura 10. Ubicación del sistema de captación en la alcaldía Cuajimalpa.

Se selecciona el intervalo de fechas desde el $1^{\circ}$ de enero de 2019 hasta el 30 de septiembre de 2019, con un total de 272 días entre las fechas mencionadas. 
2021, Instituto Mexicano de Tecnología del Agua

Open Access bajo la licencia CC BY-NC-SA 4.0

(https://creativecommons.org/licenses/by-nc-sa/4.0/)

Para el rango de fechas seleccionado se obtuvo un volumen de 33 418.24 litros (Figura 11) y 46641.42 litros (Figura 12) para el punto en Iztapalapa y Cuajimalpa, respectivamente.

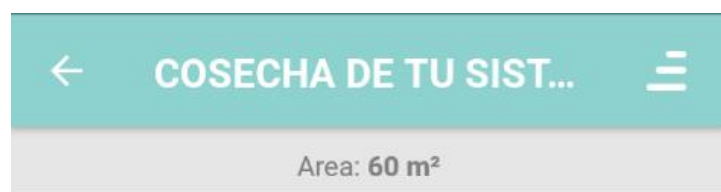

Desde el día 01/01/2019 hasta el día

30/09/2019 pudiste haber captado

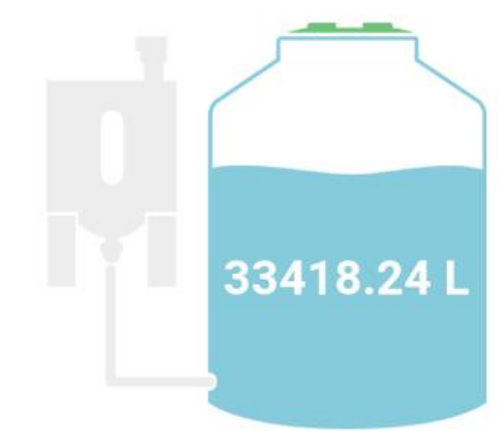

Los volúmenes mostrados son estimaciones estadísticas.

\section{CAMBIAEL RANGO DE FECHA 曲}

Figura 11. Volumen potencialmente captado en Iztapalapa. 


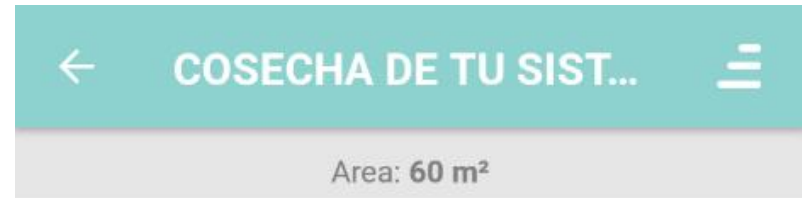

\section{Desde el día 01/01/2019 hasta el día $30 / 09 / 2019$ pudiste haber captado}

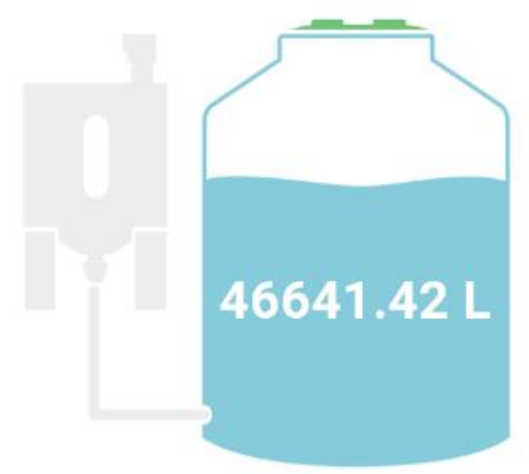

Los volúmenes mostrados son estimaciones estadísticas.

\section{CAMBIA EL RANGO DE FECHA \#曲}

Figura 12. Volumen potencialmente captado en Cuajimalpa.

\section{Discusión}


De los resultados obtenidos se observa que el volumen fue mayor para la alcaldía de Cuajimalpa, pues en las zonas sur y suroriente las precipitaciones medias anuales son mayores que para la zona nororiente (Semarnat-Conagua, 2018). En el primer caso, el volumen mostrado puede usarse en los hogares y así mermar el impacto de inundaciones urbanas. El segundo punto de análisis es importante debido a que las zonas montañosas urbanizadas provocan que las escorrentías sean de mayor velocidad y volumen, lo que provoca acumulaciones en las zonas bajas, donde el agua ya no puede escurrir.

Para el punto de estudio en Iztapalapa, el volumen significa 122.9 litros diarios para un domicilio ubicado en tal zona y considerando un promedio de 3.4 personas por vivienda (INEGI, 2015b); por lo tanto, esto equivaldría a 29.25 litros al día por habitante. Este volumen captado, si bien no complementaría el consumo promedio de la CDMX, sí representa una gran disminución durante las temporadas de lluvia, en donde se tendría menor dependencia de la red de abastecimiento pública; no obstante, en temporada de estiaje sería mayor el consumo directo de la red.

\section{Conclusiones}


La situación hídrica en el Valle de México necesita solución. En este instante, la condición no es totalmente caótica y se cuenta con tiempo para poder tomar decisiones y aplicarlas de manera correcta. Las acciones que se decidan adecuadas se deben aplicar de manera permanente y bien administradas. La colaboración entre las entidades federativas que intervienen para la toma de decisiones y la concienciación de la ciudadanía sobre el uso del agua son necesarias para dar una rápida solución a los problemas del agua.

El manejo integral del agua deberá ser indispensable. Las acciones que conlleven un uso sustentable y responsable de los recursos hídricos deberán ser adoptadas por la población (Andales et al., 2014). Es aquí donde el uso de la tecnología mediante aplicaciones móviles se deberá desarrollar, ocupar y aprovechar para tener mejores herramientas con las cuales se podrá buscar una mejor optimización del uso de los recursos con los que se cuentan.

La idea es buscar menor dependencia del agua importada a la CDMX; recuperar los acuíferos que han sido sobreexplotados en los últimos años; lograr mayor y mejor distribución del agua a lo largo de la Zona Metropolitana; disminuir las inundaciones que aquejan a los puntos más bajos de la ciudad; evitar sobrepasarla capacidad de los sistemas de drenaje; dar mayor uso a las aguas tratadas en lugar de aguas de primer uso, y prevenir a los estados aledaños incluirlos en esta situación (De Vriend et al., 2015). Podría parecer mucho trabajo por realizar, pero se cuenta con un horizonte de posibilidades para dar solución, y así evitar 
llegar a escenarios catastróficos y de lucha por un recurso tan básico para la humanidad.

\section{Referencias}

Andales, A., Bauder, T., \& Arabi, M. (2014). Mobile irrigation water managment system using a collaborative GIS and weather station. In L. Ahuja, L. Ma, \& R. Lascano (Eds.), Practical Applications of Agricultural System Models to Optimize the Use of Limited Water, Volume 5

(pp. 53-84). https://doi.org/10.2134/advagricsystmodel5.c3

Arto, I., Andreoni, V., \& Rueda-Cantuche, J. (2016). Global use of water reources: A multiregional analysis of water use, water footprint and water trade balance. Water Resources and Economics, 15,1-14. https://doi.org/10.1016/j.wre.2016.04.002

Conagua, Comisión Nacional del Agua. (2005). Sistema Cutzamala: agua para millones de mexicanos. México, DF, México: Comisión Nacional del Agua.

De Urbanisten, Deltares. (2017). Towards a water sensitive México City. Ciudad de México, México: Deltares.

De-Vriend, H. J., van Koningsveld, M., Aarninkhof, S. G., de Vries, M. B., \& Baptist, M. J. (2015). Sustainable hydraulic engineering through building with nature. Journal of Hydro-environment Research, 9(2),159-171. https://doi.org/1016/J.JHER.2014.06.004 
INEGI, Instituto Nacional de Estadística y Geografía. (2015a). Número de habitantes. Recuperado de http://cuentame.inegi.org.mx/monografias/informacion/df/poblaci on/

INEGI, Instituto Nacional de Estadística y Geografía. (2015b). Viviendas. Recuperado de http://cuentame.inegi.org.mx/monografias/informacion/df/poblaci on/vivienda. aspx?tema $=$ me $\&$ e $=09$

Isla Urbana. (2018). Isla Urbana. Recuperado de http://islaurbana.org/beneficios/

OH-II UNAM. (2016). Observatorio hidrológico. Recuperado de https://www.oh-iiunam.mx/informacion.html

OMS, Organización Mundial de la Salud. (2003). Domestic waterquantity, service level and health. Genève, Switzerland: WHO Document Production Services.

Perló-Cohen, M., \& González-Reynoso, A. E. (2005). ¿Guerra por el agua en el Valle de México?: estudio sobre las relaciones hidráulicas entre el Distrito Federal y el Estado de México. México, DF, México: PUECFundación Friedrich Ebert Stifung.

Sacmex, Sistema de Aguas de la Ciudad de México. (2017). El Agua Recurso Natural de Gran Trascendencia para la Vida. Ciudad de México: Agencia Promotor de Publicaciones.

Sedema, Secretaría del Medio Ambiente de la Ciudad de México. (2016). Cuidar el agua es cosa de tod@s. Recuperado de http://www.cuidarelagua.cdmx.gob.mx/delegacion.html 
Semarnat, Secretaría de Medio Ambiente y Recursos Naturales. (2018). Semarnat. Recuperado

de http://dgeiawf.semarnat.gob.mx:8080/ibi_apps/WFServlet?IBIF_e X=D3_AGUA01_01\&IBIC_user=dgeia_mce\&IBIC_pass=dgeia_mce $\&$ NOMBREENTIDAD $=* \&$ NOMBREANIO $=*$

Semarnat-Conagua, Secretaría de Medio Ambiente y Recursos Naturales, Comisión Nacional del Agua. (2018). Estadísticas del agua en México. Ciudad de México, México: Secretaría de Medio Ambiente y Recursos Naturales.

Shivakumar, A. R. (2017). Sustainable water supply strategy for Bangalore - A model for emerging cities in India. International Journal of Science, Technology and Society, 6(1), 6-12. https://doi.org/10.11648/j.ijsts.20180601.12

Silva, B. N., Khan, M., \& Han, K. (2018). Towards sustainable smart cities: A review of trends, architectures, components, and open challenges in smart cities. Sustainable Cities and Society, 38, 697-713. https://doi.org/10.1016/J.SCS.2018.01.053 